Original Paper http://ajol.info/index.php/ijbcs http://indexmedicus.afro.who.int

\title{
Inventaire des mauvaises herbes et des méthodes de lutte contre l'enherbement dans les exploitations de la zone cotonnière Est du Burkina Faso
}

\author{
Bétia ETIABI ${ }^{1 *}$, Bazoumana KOULIBALY ${ }^{1}$ et Hamidou TRAORE ${ }^{2}$ \\ ${ }^{I}$ Institut de l'Environnement et de Recherches Agricoles (INERA), Programme Coton, DRREA-Ouest/Farako- \\ Bâ, 01 BP 208, Bobo-Dioulasso 01, Burkina Faso. \\ ${ }^{2}$ Institut de l'Environnement et de Recherches Agricoles (INERA), Département Productions Végétales, \\ CREAF de Kamboinsé, 01 BP 476 Ouagadougou 01, Burkina Faso. \\ "Auteur correspondant ; E-mail: etiabimamadou@gmail.com; Tél: (+226) 60223779
}

\begin{tabular}{lll}
\hline Received: 08-01-2021 & Accepted: 03-08-2021 & Published: 31-08-2021 \\
\hline
\end{tabular}

\section{RESUME}

Dans la plupart des zones cotonnières du Burkina Faso, les producteurs de coton éprouvent de plus en plus des difficultés dans la gestion de l'enherbement qui induit des dommages importants au cotonnier. Cette étude a été réalisée dans la zone cotonnière Est du pays pour inventorier et connaître les principales mauvaises herbes et les méthodes de lutte des producteurs contre l'enherbement. Les paramètres évalués ont été les fréquences et les indices d'abondance-dominance, déterminés à partir des inventaires floristiques réalisés dans les champs de coton. Les espèces de mauvaises herbes nuisibles au cotonnier ont été répertoriées ainsi que les méthodes de lutte contre celles-ci. Les inventaires floristiques réalisés en 2018 et en 2019, ont permis de recenser 98 espèces de mauvaises herbes. Les dicotylédones sont prédominantes avec 80 et 91,67\% des familles de mauvaises herbes recensées respectivement, dans le Koulpélogo et le Gourma. Les analyses des fréquences et des indices d'abondance-dominance ont permis d'identifier 12 espèces de mauvaises herbes nuisibles au cotonnier. Les enquêtes ont révélé que les superficies cultivées en coton traitées aux herbicides variaient de 45,30 à $52,24 \%$ pour le glyphosate, 33,95 à 49,55\% pour le diuron et seulement $8,84 \%$ pour l'herbicide haloxyfop-Rméthyl. Des adventices comme Commelina benghalensis L., Ipomoea eriocarpa R.Br. et Rottboellia cochinchinensis (Lour.) W. Clayton ne sont pas bien maîtrisées ni par le glyphosate ni par le diuron, selon les producteurs. Le désherbage mécanique par la traction attelée, nécessite un sarclage manuel pour éliminer les mauvaises herbes présentes sur les lignes de semis du cotonnier. Le désherbage manuel est surtout pratiqué contre les mauvaises herbes non maîtrisées par le désherbage mécanique par 66,70 à 70,70\% des producteurs. Au regard des limites des trois méthodes de lutte (chimique, mécanique et manuel) l'étude suggère leur combinaison pour une meilleure maîtrise de l'enherbement du cotonnier.

(C) 2021 International Formulae Group. All rights reserved.

Mots clés : Zone cotonnière, mauvaises herbes, relevé floristique, inventaire, désherbage chimique. 


\title{
Inventory of weeds and the control methods on farms in the eastern cotton growing area of Burkina Faso
}

\begin{abstract}
In most cotton growing areas in Burkina Faso, cotton farmers are experiencing greater difficulties with weeds, which cause significant damage to cotton yield. This study has been conducted in the eastern cotton growing area of the country to inventory the main weeds and the methods of weed control implemented by farmers. The evaluated parameters were the frequencies and the abundance-dominance indices, determined from floristic inventories in cotton fields. The main weed species and their control methods have been identified in cotton fields. The results showed that 98 weeds species were identified in cotton fields in 2018 and 2019. Dicotyledons are predominant with $80 \%$ and $91.67 \%$ of weed families identified respectively, in the provinces of Koulpélogo and Gourma. Frequency and abundance-dominance indices analysis revealed 12 weeds species causing damage to cotton. Surveys revealed that cotton surfaces treated with herbicides vary from 45.30 to $52.24 \%$ for glyphosate, 33.95 to $49.55 \%$ for diuron and only $8.84 \%$ for post-emergence herbicide haloxyfop-Rmethyl. According to the farmers, glyphosate or diuron-based herbicides are less efficient against weeds such as Commelina benghalensis L., Ipomoea eriocarpa R.Br. and Rottboellia cochinchinensis (Lour.) W. Clayton. Mechanical weed control by animal traction requires an additional hand weeding to eliminate weeds on the cotton seedling rows. Hand weeding is mainly practiced against the uncontrolled weeds by mechanical weeding by $66.70 \%$ to $70.70 \%$ of farmers. Considering the limits of the three control methods (chemical, mechanical and hand weeding) this study suggests their combination for a better weed control in cotton fields.
\end{abstract}

(C) 2021 International Formulae Group. All rights reserved.

Keywords: Cotton, weeds, inventory, floristic record, chemical weeding.

\section{INTRODUCTION}

Les mauvaises herbes constituent l'une des principales contraintes biologiques (Oerke, 2006) qui affectent les productions agricoles mondiales et plus particulièrement celles des pays en voie de développement (Marnotte et Le Bourgeois, 2002). En effet, l'enherbement des exploitations agricoles est considéré par de nombreux auteurs comme étant à l'origine des principales causes des pertes de rendements au champ (Oerke, 2006 ; Touré et al., 2008 ; Boudjedjou, 2010 ; Manalil et al., 2017 ; Ahonon et al., 2018). Des baisses de rendements de $13,8 \%$ à $90 \%$ dues aux dommages des mauvaises herbes sur les cultures, ont été rapportées par plusieurs auteurs (Oerke, 2006 ; Cardoso et al., 2011 ; Manalil et al., 2017 ; Gharde et al., 2018).

Dans les systèmes de production cotoncéréales, le cotonnier est souvent plus confronté que les autres cultures, aux effets néfastes de la concurrence des mauvaises herbes. Les pertes de rendement sur le cotonnier dues aux mauvaises herbes sont d'environ $35 \%$ en absence de désherbage pendant les 35 premiers jours après les semis (Marnotte et Le Bourgeois, 2002; Oerke, 2006). Au Burkina Faso, la production cotonnière fait l'objet d'une baisse considérable des rendements ces dernières années imputables à plusieurs facteurs dont l'enherbement. Dans la zone cotonnière de l'Est du pays, l'insuffisance de la main d'œuvre pour le désherbage et l'utilisation anarchique des herbicides, ont engendré un enherbement important des champs rendant préoccupante la question de la gestion des mauvaises herbes. Les producteurs de coton dans cette zone ont de plus en plus des difficultés pour maitriser l'enherbement dans leurs exploitations. Pourtant, une mauvaise gestion des mauvaises herbes peut entraîner jusqu'à 90\% des pertes de rendement du cotonnier (Manalil et al., 2017). Ainsi, la réduction des contraintes de production de coton due à l'enherbement passe par une connaissance de la composition de la flore des mauvaises herbes qui est indispensable à toute amélioration des techniques de lutte contre l'enherbement (Karar et al., 2005 ; Ferrell et 
al., 2020). La plupart des études réalisées au Burkina Faso sur les mauvaises herbes ont été effectués sur des cultures céréalières (Yonli et al., 2006 ; Yonli et al., 2010 ; Boussim et al., 2011 ; Garane et al., 2019).

La présente étude a eu pour objectif d'identifier les mauvaises herbes dommageables au cotonnier et de déterminer les principales contraintes dans la gestion de l'enherbement dans les exploitations de la zone cotonnière Est du Burkina Faso.

\section{MATERIEL ET METHODES \\ Sites de l'étude}

Cette étude a été réalisée dans les provinces du Koulpélogo et du Gourma dans l'Est du Burkina Faso, dans la zone d'intervention de la Société Cotonnière du Gourma (SOCOMA), qui est l'une des trois sociétés cotonnières du pays (Figure 1). Les coordonnées géographiques des sites d'étude sont présentées dans le Tableau 1. Il s'agit de 11 villages dont cinq sont localisés dans la Province du Koulpélogo et les six autres dans la Province du Gourma. Ces sites d'étude se trouvent dans la zone agro-écologique Soudano-sahélienne qui possède un climat tropical de savane. Cette zone est marquée par une saison pluvieuse de 3 à 4 mois, et une longue saison sèche qui dure environ 8 à 9 mois, et qui est subdivisée en une saison sèche froide (octobre à mars), suivie d'une saison sèche et chaude (mars à juin). La moyenne pluviométrique annuelle est comprise entre 700 $\mathrm{mm}$ et $900 \mathrm{~mm}$. La pluviométrie moyenne annuelle des 33 dernières années est de 809,33 $\mathrm{mm}$. Les minimas de température s'observent entre décembre et janvier avec $17{ }^{\circ} \mathrm{C}$, tandis que les maxima de température sont obtenus en avril et mai avec $40{ }^{\circ} \mathrm{C}$ (MEEVCC, 2018). Le sorgho, le mil, le maïs, le coton, le niébé et le sésame, représentent les cultures dominantes rencontrées dans les exploitations agricoles des producteurs.

\section{Méthodes}

Pendant deux années, en 2018 et 2019, des enquêtes ont été conduites dans les exploitations cotonnières de la zone SOCOMA afin de recenser les principales mauvaises herbes et de cerner les contraintes et les méthodes de gestion de l'enherbement par les producteurs. Le questionnaire élaboré pour ces enquêtes a porté d'une part, sur l'inventaire des mauvaises herbes rencontrées dans les exploitations et, d'autre part, sur les contraintes et stratégies de gestion de l'enherbement.

\section{Inventaire des mauvaises herbes}

La méthode des relevés floristiques adoptée a été « le tour du champ » pour les inventaires des mauvaises herbes dans les champs de coton. Cette méthode consiste à parcourir le champ dans toutes les directions afin de recenser de façon systématique, toutes les espèces de mauvaises herbes présentes. Dans chaque champ de cotonnier enquêté, les inventaires des mauvaises herbes ont été réalisés sur une superficie n'excédant pas 0,25 ha qui est considérée comme une unité relativement homogène par Le Bourgeois (1993). L'abondance-dominance de chaque espèce de mauvaise herbe a été estimée à partir de l'échelle modifiée de Braun-Blanquet (Le Bourgeois, 1993) :

1 = individus peu abondants ou abondants, mais à recouvrement faible ;

$2=$ individus très abondants ou recouvrant $1 / 20$ de la surface ;

$3=$ individus recouvrant de $1 / 4$ à $1 / 2$ de la surface, abondance quelconque ;

$4=$ individus recouvrant de $1 / 2$ à $3 / 4$ de la surface, abondance quelconque ;

$5=$ individus recouvrant plus des $3 / 4$ de la surface, abondance quelconque.

$\mathrm{Au}$ cours de cette étude, 30 relevés floristiques ont été effectués en 2018 dans la province du Koulpélogo contre 82 en 2019 , dans la province du Gourma, soit un total de 112 relevés floristiques à raison d'un seul relevé par producteur.

Diagnostic des contraintes de gestion des mauvaises herbes

L'échantillon des 112 producteurs retenus pour les inventaires floristiques a également été soumis à un questionnaire pour les méthodes de lutte pratiquées contre les mauvaises herbes; le questionnaire a permis de s'imprégner des contraintes liées à la gestion de l'enherbement des parcelles cotonnières. 


\section{Traitement des données}

Les données recueillies sur l'enherbement des champs ont été soumises à une analyse quantitative qui s'est basée sur la fréquence centésimale et l'abondancedominance pour montrer l'importance agronomique des espèces inventoriées (Lebreton et Le Bourgeois, 2005). La fréquence absolue (Fa) de l'espèce ou fréquence spécifique est le nombre de fois (n) qu'une espèce (e) a été observée dans $(\mathrm{N})$ relevés lors d'un échantillonnage. La fréquence relative ou fréquence centésimale $(\mathrm{Fc})$ de l'espèce est le rapport de la fréquence absolue sur le nombre total de relevés déterminée par la formule 1 ci-dessous :
$\mathrm{Fa}(\mathrm{e})$

$$
\mathrm{Fc}=---------\mathrm{x} 100
$$

L'indice d'abondance-dominance moyenne de l'espèce (e) est la somme des notes d'abondance-dominance sur le nombre total de relevés $(\mathrm{N})$ où l'espèce est présente. Cet indice est calculé selon la formule 2 suivante :

$$
\text { I-ADmoy =----------------- }
$$

Les données recueillies sur les méthodes de lutte contre les mauvaises herbes ont été analysées par le logiciel Sphinx plus ${ }^{2}$ version 5.0.

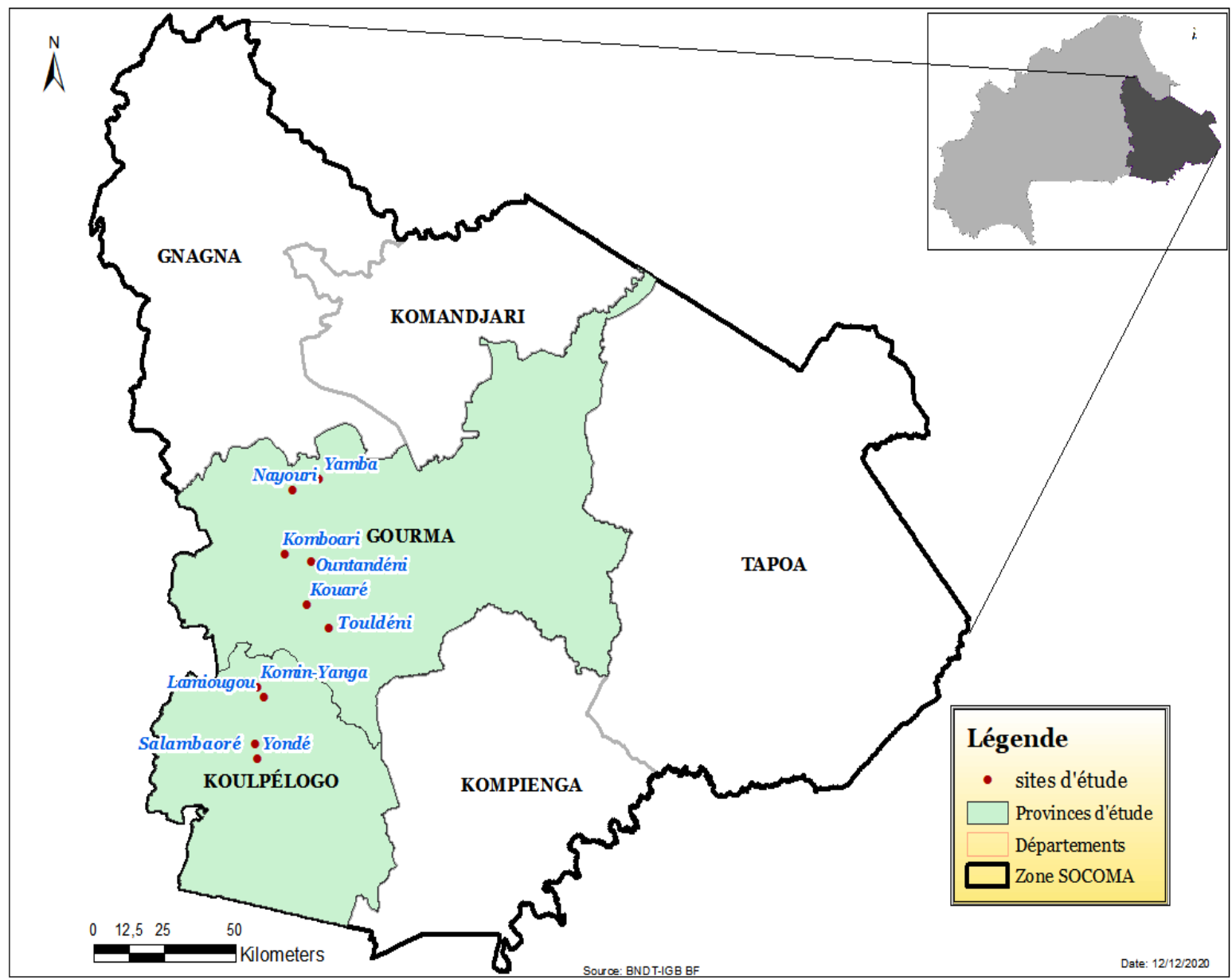

Figure 1 : Localisation des provinces du Gourma et du Koulpélogo dans la zone cotonnière Est du Burkina Faso. 
Tableau 1 : Coordonnées géographiques des sites d'étude.

\begin{tabular}{|c|c|c|c|}
\hline \multirow[t]{2}{*}{ Provinces } & \multirow[t]{2}{*}{ Villages } & \multicolumn{2}{|c|}{ Coordonnées géographiques } \\
\hline & & Latitude & Longitude \\
\hline \multirow[t]{6}{*}{ Gourma } & Kouaré & $11^{\circ} 56^{\prime} 28.49^{\prime \prime} \mathrm{N}$ & $0^{\circ} 17^{\prime} 53.43^{\prime \prime} \mathrm{E}$ \\
\hline & Touldéni & $11^{\circ} 51^{\prime} 11.26^{\prime \prime} \mathrm{N}$ & $0^{\circ} 21 ' 25.64 " \mathrm{E}$ \\
\hline & Ountandéni & $12^{\circ} 3^{\prime} 29.69^{\prime \prime} \mathrm{N}$ & $0^{\circ} 17^{\prime} 36.25 " \mathrm{~B}$ \\
\hline & Comboari & $12^{\circ} 4^{\prime} 45.23^{\prime \prime} \mathrm{N}$ & $0^{\circ} 14^{\prime} 43.21^{\prime \prime} \mathrm{E}$ \\
\hline & Yamba & $12^{\circ} 18^{\prime} 5.11^{\prime \prime} \mathrm{N}$ & $0^{\circ} 20^{\prime} 19.15^{\prime \prime} \mathrm{E}$ \\
\hline & Nayouri & $12^{\circ} 16^{\prime} 27.89^{\prime \prime} \mathrm{N}$ & $0^{\circ} 14^{\prime} 44.82^{\prime \prime} \mathrm{E}$ \\
\hline \multirow[t]{5}{*}{ Koulpélogo } & Comin-yanga & $11^{\circ} 42^{\prime} 28.75^{\prime \prime} \mathrm{N}$ & $0^{\circ} 08^{\prime} 19.43^{\prime \prime} \mathrm{E}$ \\
\hline & Lamiougou & $11^{\circ} 40^{\prime} 37.9^{\prime \prime} \mathrm{N}$ & $0^{\circ} 09^{\prime} 44.34 \mathrm{"E}$ \\
\hline & Bousgou & $11^{\circ} 33^{\prime} 8.96$ "N & $0^{\circ} 06^{\prime} 30.83$ "E \\
\hline & Salambaoré & $11^{\circ} 32^{\prime} 24.66^{\prime \prime} \mathrm{N}$ & $0^{\circ} 06{ }^{\prime} 59.91 " \mathrm{E}$ \\
\hline & Yondé & $11^{\circ} 30^{\prime} 04.95^{\prime \prime} \mathrm{N}$ & $0^{\circ} 08^{\prime} 25.33^{\prime \prime} \mathrm{E}$ \\
\hline
\end{tabular}

\section{RESULTATS}

\section{Composition floristique des mauvaises herbes inventoriées}

Les inventaires réalisés dans les sites d'étude au cours des deux années d'étude ont permis de recenser au total 98 espèces de mauvaises herbes réparties en 65 genres et 24 familles (Figure 2). Dans les exploitations cotonnières des cinq sites du Koulpélogo, 70 espèces de mauvaises herbes appartenant à 20 familles ont été identifiées contre 87 espèces appartenant à 24 familles dans les sites du Gourma (Figure 2). Les dicotylédones ont été prédominantes avec 80 et $91,67 \%$ des familles de mauvaises herbes recensées, respectivement, dans le Koulpélogo et le Gourma. Dans les exploitations des sites du Koulpélogo, on constate que les mauvaises herbes les plus rencontrées se regroupent dans 7 familles que sont les Poaceae $(27,14 \%)$, les Asteraceae (8,57\%), les Fabaceae $(7,14 \%)$, les Rubiaceae $(7,14 \%)$, les Malvaceae $(7,14 \%)$, les Cyperaceae $(5,71 \%)$, et les Commelinaceae $(5,71 \%)$. En revanche, dans les exploitations des sites du Gourma, les 7 familles les plus représentées sont les Poaceae $(22,98 \%)$, les Amaranthaceae $(11,49 \%)$, les Fabaceae $(8,04 \%)$, les Cyperaceae $(6,89 \%)$, les Malvaceae $(5,74 \%)$, Commelinaceae $(5,74 \%)$ et les Asteraceae (5,74\%). Dans l'ensemble, il ressort que ces familles prédominantes des mauvaises herbes, renferment à elles seules, 67,14 et $71,26 \%$ des espèces inventoriées respectivement, dans le Koulpélogo et dans le Gourma.

\section{Nuisibilité des mauvaises herbes}

La distribution des espèces de mauvaises herbes en fonction des fréquences et des indices d'abondance-dominance a permis d'identifier les mauvaises herbes nuisibles dans les exploitations cotonnières (Tableau 2). Les mauvaises herbes dont la fréquence est supérieure $50 \%$ et l'indice d'abondancedominance supérieur à 1,5 sont considérées comme étant nuisibles au cotonnier. Les analyses des fréquences ont permis d'identifier 17 espèces de mauvaises herbes dont les fréquences sont supérieures à 50\% dans les exploitations enquêtées (Tableau 2). D'autre 
part, il a été recensé 14 espèces de mauvaises herbes dans le Koulpélogo et 10 dans le Gourma dont les indices moyens d'abondancedominance sont supérieurs à 1,5 (Tableau 3). Les mauvaises herbes inventoriées ayant à la fois une fréquence supérieure à $50 \%$ et un indice moyen d'abondance-dominance supérieur à 1,5 sont aux nombres de 9 et 12 , respectivement dans le Gourma et le Koulpélogo. Ainsi, les mauvaises herbes potentiellement nuisibles au cotonnier et recensées à la fois dans l'ensemble des deux Provinces d'étude sont Ipomoea eriocarpa $\mathrm{R}$. Br., Hyptis spicigera Lam., Digitaria horizontalis Willd., Kyllinga squamulata Thonn., Leucas martinicensis Jacq., Spermacoce stachydea D.C., Mitracarpus villosus SW., Commelina benghalensis L. et Rottboellia cochinchinensis (Lour.) W. Clayton.

\section{Méthodes de lutte contre les mauvaises herbes}

L'étude a révélé que la plupart des producteurs enquêtés pratiquent le désherbage chimique à l'aide de trois types d'herbicides. Il s'agit d'un herbicide non sélectif à base de glyphosate, le KALACH 360 SL (360 g/l de glyphosate), d'un herbicide de prélevée à base de diuron, le DIURALM 80 WG $(800 \mathrm{~g} / \mathrm{kg}$ de diuron) et d'un herbicide de post-levée du cotonnier à base d'haloxyfop-R-méthyl, le GRAMI 108 EC (108 g/l de Haloxyfop-Rméthyl), tous fournis à crédit aux producteurs par la SOCOMA. Cependant, les herbicides ne sont pas appliqués par les producteurs sur toutes les superficies cultivées en coton (Figure 3). Ainsi, les enquêtes ont montré qu'en 2018 dans les sites du Koulpélogo, 52,24\% des superficies en coton ont été traités avec le glyphosate contre $49,55 \%$ des superficies traitées avec le diuron alors qu'aucune application d'herbicide de post-levée (haloxyfop-R-méthyl) n'a été notée. Dans les sites du Gourma en 2019, les superficies cultivées en coton et traitées avec le glyphosate, le diuron et l'haloxyfop-R-méthyl étaient de $45,30 \%, 33,95 \%$ et $8,84 \%$, respectivement (Figure 3). En évoquant les limites de la lutte chimique, les producteurs ont souligné que certaines mauvaises herbes telles que Commelina benghalensis L., Ipomoea eriocarpa R.Br. et Rottboellia cochinchinensis (Lour.) W. Clayton ne sont pas maîtrisées par le glyphosate (Figure 4) et par le diuron (Figure 5). Les enquêtes ont aussi révélé que pendant les semis du cotonnier, certains producteurs appliquent des mélanges d'herbicides à base de glyphosate et de diuron pour mieux maîtriser les mauvaises herbes susceptibles de concurrencer les cotonniers à la levée.

En ce qui concerne le désherbage mécanique en complément au désherbage chimique, des différences statistiques ont été notées entre les sites d'étude pour les équipements agricoles et les animaux de trait (Tableau 4). Dans les sites d'étude du Gourma, les niveaux d'équipement sont significativement supérieurs à ceux des sites du Koulpélogo, pour le nombre moyen de triangles sarcleurs $(p \leq 0,0186)$, de tracteurs ( $p$ $\leq 0,0001)$ de rayonneurs $(\mathrm{p} \leq 0,0001)$ et de charrues bovines $(\mathrm{p} \leq 0,0397)$. Les outils aratoires les plus utilisés pour le désherbage mécanique sont la charrue bovine et la charrue asine servant à la préparation du sol et au buttage, puis le triangle sarcleur. Le désherbage mécanique généralement réalisé en traction attelée, ne permet pas toutefois de maîtriser les mauvaises herbes présentes sur les lignes de semis du cotonnier, ce qui nécessite alors un sarclage manuel complémentaire.

Dans les sites d'étude, les méthodes de lutte chimique et mécanique sont le plus souvent accompagnées du désherbage manuel qui vise à éliminer, par l'arrachage manuel, certaines mauvaises herbes non maîtrisées par les herbicides et par les différents désherbages mécaniques (Figure 6). Le constat final est que le désherbage manuel est surtout pratiqué contre les mauvaises herbes non maîtrisées par le désherbage mécanique par 66,70 et $70,70 \%$ des exploitations, respectivement dans le Koulpélogo et le Gourma. Ce type de désherbage exige plus de temps de travail et nécessite de recourir à une importante main d'œuvre, non disponible dans les exploitations cotonnières. 


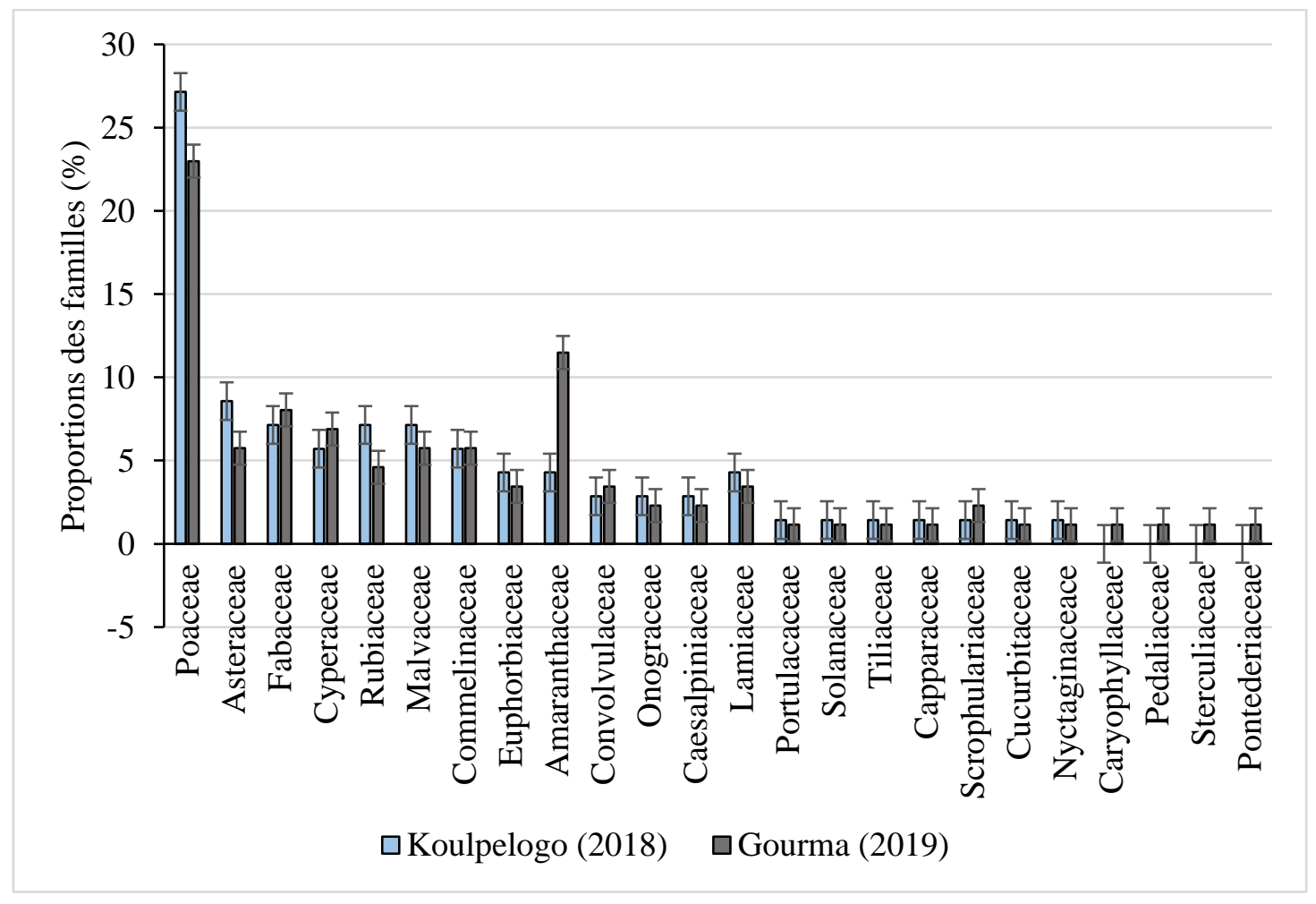

Figure 2 : Familles des mauvaises herbes inventoriées dans les champs de cotonnier des sites d'étude.

Tableau 2 : Principales mauvaises herbes dont la fréquence de relevé dans les champs de cotonnier est supérieure à $50 \%$.

\begin{tabular}{|c|c|c|c|c|}
\hline & & & \multicolumn{2}{|c|}{ Province } \\
\hline \multirow[t]{2}{*}{ Classes } & \multirow[t]{2}{*}{ Familles } & \multirow[t]{2}{*}{ Espèces de mauvaises herbes } & $\begin{array}{l}\text { Koulpélogo } \\
\text { (2018) }\end{array}$ & $\begin{array}{l}\text { Gourma } \\
\text { (2019) }\end{array}$ \\
\hline & & & \multicolumn{2}{|c|}{ Fréquences $(\%)$} \\
\hline \multirow{11}{*}{ Monocotylédones } & \multirow{8}{*}{ Poaceae } & Digitaria horizontalis Willd. & 90 & 92,68 \\
\hline & & $\begin{array}{l}\text { Rottboellia cochinchinensis (Lour.) W. } \\
\text { Clayton }\end{array}$ & 66,66 & 70,73 \\
\hline & & Eragrostis tremula Hochst. & 63,34 & 62,19 \\
\hline & & Pennisetum pedicellatum Trin. & 60 & 53,65 \\
\hline & & Dactyloctenium aegyptium (L.) P. Beauv. & 53,33 & 62,19 \\
\hline & & $\begin{array}{l}\text { Setaria pallide-fusca (Schum.) Stapf \& } \\
\text { CE }\end{array}$ & - & 60,97 \\
\hline & & Eragrostis ciliaris (L.) R.Br. & - & 59,75 \\
\hline & & Brachiaria lata (Schum.) C. E. Hubb. & - & 51,21 \\
\hline & Cyperaceae & Kyllinga squamulata Thonn. & 83,34 & 91,46 \\
\hline & Commelinaceae & Commelina benghalensis L. & 66,66 & 63,41 \\
\hline & & Commelina forskalaei Valh. & - & 56,09 \\
\hline \multirow{3}{*}{ Dicotylédones } & Convolvulaceae & Ipomoea eriocarpa $\mathrm{R} . \mathrm{Br}$. & 100 & 90,24 \\
\hline & Lamiaceae & Hyptis spicigera Lam. & 96,67 & 80,48 \\
\hline & & Leucas martinicensis (Jacq.) Ait. f. & 80 & 73,17 \\
\hline
\end{tabular}




\begin{tabular}{llll}
\hline Tiliaceae & Corchorus tridens L. & 86,67 & 64,63 \\
\hline Rubiaceae & Spermacoce stachydea D.C. & 76,67 & 63,41 \\
& Mitracarpus villosus (SW.) & 73,34 & 76,82 \\
\hline Fabaceae & Alysicarpus ovalifolius (Schum. et & 73,34 & - \\
& Thonn.) L & & \\
\hline Amaranthaceae & Amaranthus viridis L. & 66,67 & - \\
\hline Euphorbiaceae & Phyllanthus amarus Schum. \& Thon & 63,33 & - \\
\hline Asteraceae & Acanthospermum hispidium D.C. & 56,67 & - \\
\hline
\end{tabular}

Tableau 3 : Mauvaises herbes dont l'indice moyen d'abondance-dominance (I-AD) est supérieur à 1,5 dans les sites d'étude.

\section{Province}

\begin{tabular}{lcc}
\multirow{2}{*}{ Espèces de mauvaises herbes } & Koulpélogo (2018) & Gourma (2019) \\
\cline { 2 - 3 } & \multicolumn{2}{c}{ I-AD } \\
\hline Digitaria horizontalis Willd & 2,30 & 1,96 \\
Spermacoce stachydea D.C. & 2,13 & 1,66 \\
Spermacoce chaetocephala D.C. & 2,00 & - \\
Kyllinga squamulata Thonn. & 1,99 & 1,95 \\
Eragrostis tremula Hochst. & 1,99 & - \\
Hyptis spicigera Lam. & 1,97 & 1,81 \\
Leucas martinicensis (Jacq.) Ait. f. & 1,81 & 1,61 \\
Spermacoce ruelliae D.C. & 1,78 & - \\
Commelina benghalensis L. & 1,74 & 1,82 \\
Rottboellia cochinchinensis (Lour.) W. Clayton & 1,71 & 1,54 \\
Corchorus tridens L. & 1,63 & - \\
Ipomoea eriocarpa R. Br & 1,57 & 1,72 \\
Dactyloctenium aegyptium (L.) P. Beauv & 1,53 & - \\
Mitracarpus villosus SW. & 1,51 & 1,78 \\
Kyllinga bulbosa Beauv. & - & 1,52 \\
\hline
\end{tabular}

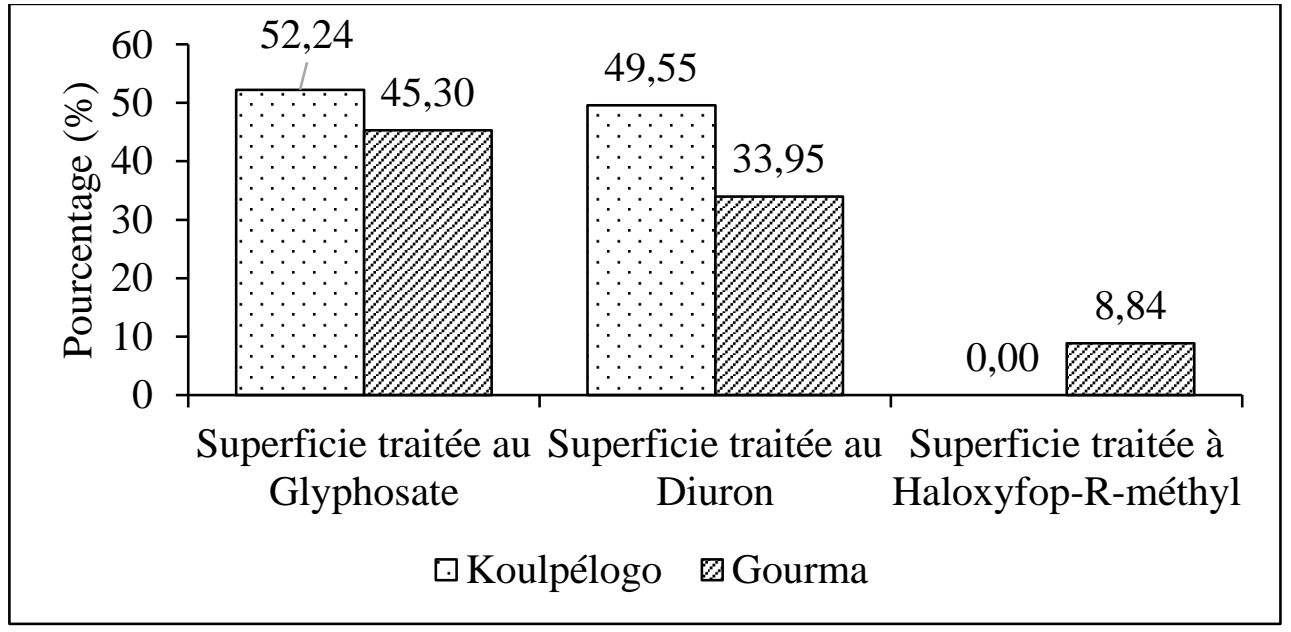

Figure 3 : Superficies traitées aux herbicides dans le Koulpélogo et dans le Gourma. 


\section{Proportions des producteurs}

\section{Cassia obtusifolia L.}

Corchorus tridens $L$.

Commelina forskalei Valh.

Sida acuta Burm.f.

Cyperus rotundus $L$

Alysicarpus ovalifolius (Schum. et

Acanthospermum hispidium DC

Ipomea eriocarpa $R$. Br.

Dactyloctenium aegyptium (L.) P. Beauv

Rottboellia cochinchinensis (Lour.) W.

Commelina benghalensis $L$

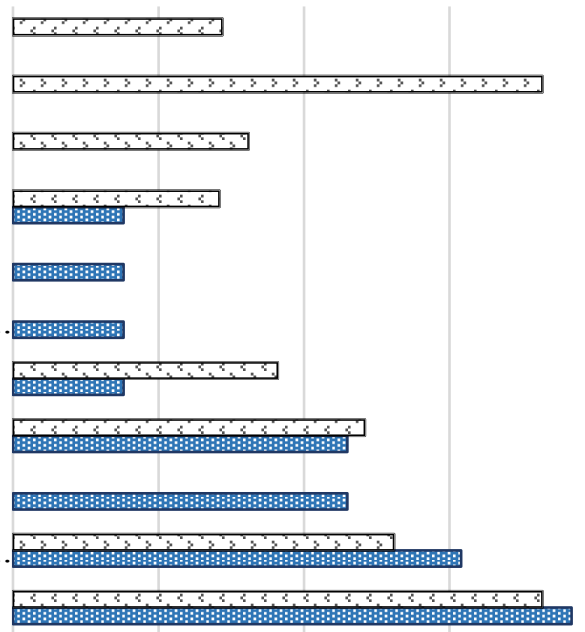

$0 \% \quad 5 \% \quad 10 \% \quad 15 \% \quad 20 \%$

口Gourma (2019) 圈 Koulpélopgo (2018)

Figure 4: Mauvaises herbes non maîtrisées par les herbicides à base de glyphosate selon les producteurs.

\section{Proportions des produteurs}

Cassia obtusifolia L.

Sida acuta Burm. $f$.

Commelina benghalensis $L$

Brachiaria deflexa (Schum.) C. E. Hubb

Acanthospermum hispidium DC

Rottboellia cochinchinensis (Lour.) W. Clayton

Spermacoce ruelliae D.C

Spermacoce stachydea D.C.

Mitracarpus villosus (SW.)

Corchorus tridens $L$.

Kyllinga squamulata Thonn.

Hyptis spicigera Lam.

Ipomea eriocarpa $R$. Br.

Dactyloctenium aegyptium (L.) P. Beauv

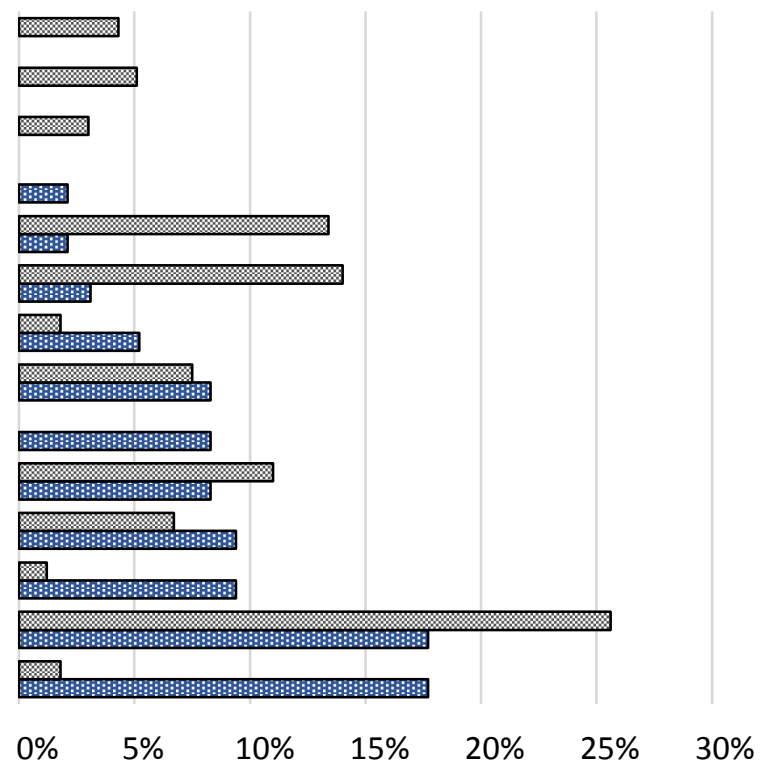

- Gourma (2019) 四 Koulpélopgo (2018)

Figure 5 : Mauvaises herbes non maîtrisées par les herbicides à base de diuron selon les producteurs. 
Tableau 4 : Nombre d'équipements agricoles et d'animaux de trait par exploitation dans les sites d'étude.

\begin{tabular}{llcccccc}
\hline \multirow{2}{*}{ Province } & $\begin{array}{c}\text { Charrue } \\
\text { bovine }\end{array}$ & $\begin{array}{c}\text { Charrue } \\
\text { Asine }\end{array}$ & Rayonneur & $\begin{array}{c}\text { Triangle } \\
\text { Sarcleur }\end{array}$ & Tracteur & $\begin{array}{l}\text { Boufs de } \\
\text { trait }\end{array}$ & $\begin{array}{l}\text { Anes } \\
\text { de trait }\end{array}$ \\
\cline { 2 - 8 } & \multicolumn{7}{c}{ Nombre/exploitation } \\
\hline Koulpélogo & $1,69 \mathrm{a}$ & $0,33 \mathrm{a}$ & $1,33 \mathrm{a}$ & $1,19 \mathrm{a}$ & $1,33 \mathrm{a}$ & $2,73 \mathrm{a}$ & $1,36 \mathrm{a}$ \\
Gourma & $1,32 \mathrm{~b}$ & $0,26 \mathrm{a}$ & $0,10 \mathrm{~b}$ & $0,90 \mathrm{~b}$ & $0,00 \mathrm{~b}$ & $2,52 \mathrm{a}$ & $1,07 \mathrm{a}$ \\
\hline $\mathrm{F}$ & 4,24 & 0,08 & 185,7 & 5,62 & 160,14 & 0,65 & 2,46 \\
Probabilité (5\%) & 0,0397 & 0,07715 & 0,0001 & 0,0186 & 0,0001 & 0,4281 & 0,1156
\end{tabular}

Dans la même colonne, les valeurs suivies d'une même lettre ne sont pas différentes significativement selon le test de Fisher au seuil de probabilité de $5 \%$.

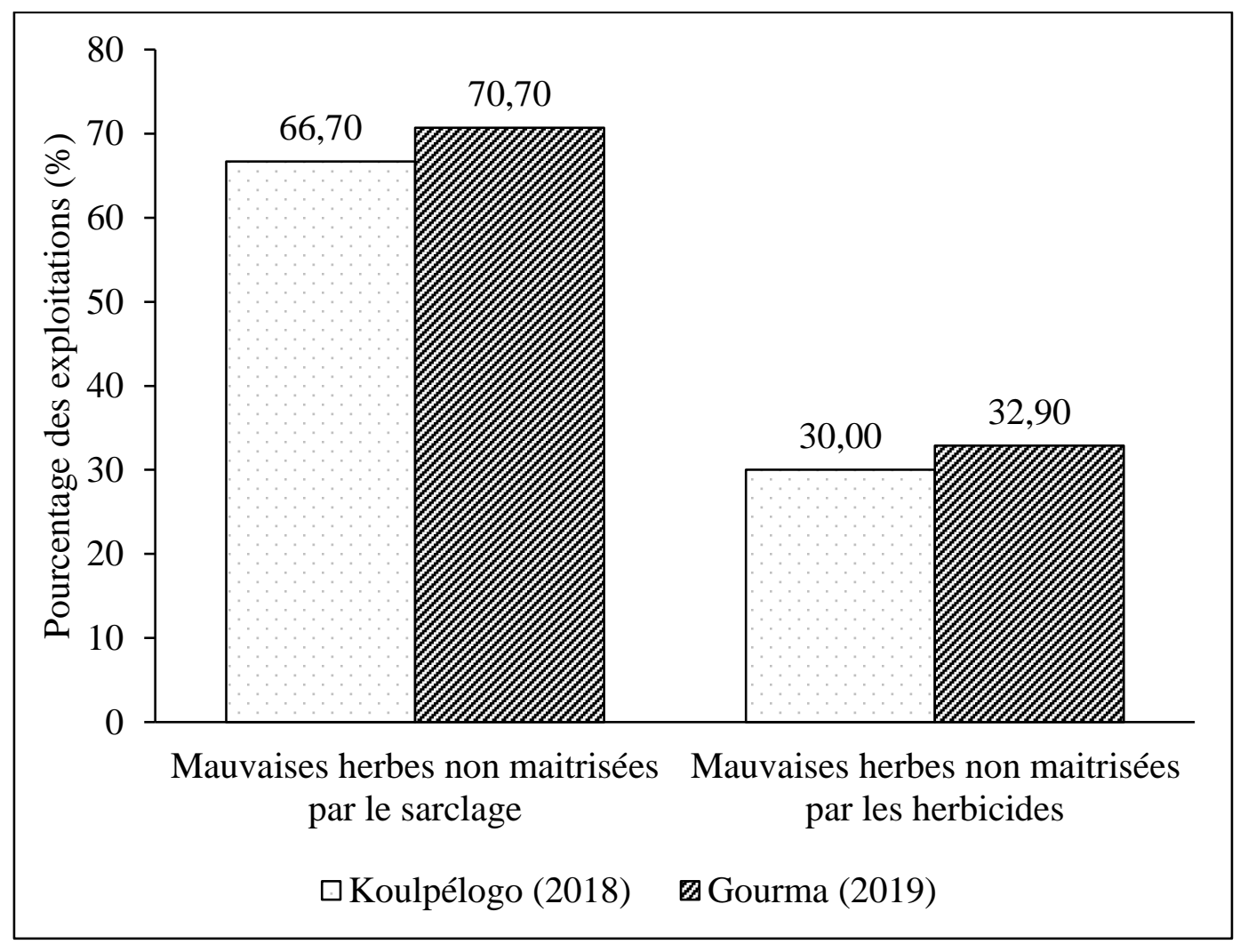

Figure 6 : Types de mauvaises herbes ciblées par le désherbage manuel. 


\section{DISCUSSION}

\section{Diversité et nuisibilité des mauvaises herbes}

Les nombres importants de familles et d'espèces de mauvaises herbes recensées dénotent une bonne diversité floristique dans les exploitations cotonnières de l'Est du Burkina Faso qui est similaire à celle de la zone cotonnière sud du Bénin décrite par Ahanchédé (1997). Pourtant, cette flore est considérée comme étant moins riche par rapport celle établie par Diouf et al. (2020) qui ont recensé 204 espèces de mauvaises herbes réparties dans 118 genres et 35 familles dans le bassin cotonnier du Sénégal. Cette flore adventice est également moins riche comparativement aux champs de sorgho au Sénégal où 232 espèces d'adventices ont été recensées par $\mathrm{Ka}$ et al. (2019). Cette plus faible diversité floristique notée pourrait s'expliquer par le fait que la présente étude n'a concerné qu'une partie de la zone cotonnière de l'Est, et d'autre part, elle s'est surtout intéressée aux principales mauvaises herbes potentiellement nuisibles et difficiles à maîtriser par les producteurs. Les familles dominantes des mauvaises herbes inventoriées dans nos sites d'étude, notamment les Poaceae, Asteraceae, Fabaceae, Malvaceae, Cyperaceae, Rubiaceae, Amaranthaceae et Commelinaceae figurent parmi celles qui ont été également répertoriées au Sénégal par Ka et al. (2019) dans les champs de sorgho et par Diouf et al. (2020), dans le bassin cotonnier. Ces familles prédominantes renfermeraient un nombre élevé d'espèces de mauvaises herbes à cause de la capacité de ces espèces à proliférer dans plusieurs milieux (Fried, 2010).

$\mathrm{Au}$ total, 12 espèces de mauvaises herbes potentiellement nuisibles au cotonnier ont été identifiées dans les zones d'étude, avec une prédominance des dicotylédones suivies des graminées. Dans leurs travaux, Traoré et Maillet (1992) avaient montré que ces mauvaises herbes nuisibles imposaient de fortes concurrences aux cotonniers et se distinguaient des autres, selon Ahonon et al. (2018), par leur fréquence élevée (> 50\%) et par leur indice d'abondance-dominance supérieur à 1,5. Leur nuisibilité serait due à une production importante de semences, des levées persistantes, des pics de germination étalés durant le cycle cultural du cotonnier et une capacité à infester, en fortes colonies, les milieux où elles se développent (Le Bourgeois, 1993 ; Bassene et al., 2012 ; Diouf et al., 2020). Par ailleurs, la plupart de ces espèces ont déjà été signalées dans divers pays, comme étant nuisibles à d'autres cultures comme le sorgho, le maïs et le mil (Traoré et Maillet, 1998 ; Bassene et al., 2012 ; Ka et al., 2020).

\section{Efficacité des moyens de lutte contre l'enherbement}

S'agissant de la lutte contre les mauvaises herbes, la majorité des producteurs ont recours à la lutte chimique, surtout au moyen d'un herbicide de prélevée à base de diuron et d'un herbicide non sélectif à base de glyphosate. Ces deux types d'herbicides ne sont appliqués qu'au moment des semis. Pourtant, la plupart des mauvaises herbes nuisibles inventoriées ont une germination étalée dans le temps. De ce fait, l'utilisation des herbicides de prélevée ne protège que temporairement le cotonnier et n'empêche pas la reproduction des mauvaises herbes qui, d'après Ahonon et al. (2018), n'intervient qu'en fin de cycle. L'herbicide de post-levée (à base d'haloxyfop-R-méthyl), qui est très peu appliqué par les producteurs dans la zone cotonnière Est, n'est efficace que sur les graminées et, de ce fait, ne permet pas de maîtriser les dicotylédones qui sont pourtant abondantes et nuisibles au cotonnier (Traoré et Maillet, 1998). Ces résultats dénotent les limites de la lutte chimique rapportées par Koulibaly et al. (1998) et suggèrent alors une bonne combinaison des différents types d'herbicides pour mieux maîtriser l'enherbement du cotonnier. Par ailleurs, le désherbage mécanique pratiqué pour limiter l'infestation des parcelles par les mauvaises herbes, nécessite l'utilisation d'un corps sarcleur en traction animale. Ce désherbage attelé exige un sarclage complémentaire à la daba pour l'entretien des lignes de semis du cotonnier, toute chose qui augmente le temps de travail et le coût du désherbage d'après Olina-Bassala et al. (2010). Dans tous les cas, certaines espèces de mauvaises herbes comme 
Digitaria horizontalis et Commelina benghalensis repoussent facilement (Le Bourgeois et Marnotte, 1994; Bassene et al., 2012). Selon Le Bourgeois (1993), la phase germinative d'Ipomoea eriocarpa n'est pas affectée par les travaux du sol qui favorisent également la germination de Commelina benghalensis et Hyptis spicigera. En plus, Kyllinga squamulata est difficile à éliminer lorsque ses racines sont couvertes de sol (Bassene et al., 2012). Le désherbage manuel pratiqué surtout vers la fin du cycle du cotonnier permet d'éliminer les mauvaises herbes susceptibles de gêner la récolte du coton sans pour autant réduire la production des semences des mauvaises herbes déjà en maturité. Nos enquêtes ont mis en exergue une combinaison par les producteurs des différentes méthodes de lutte contre l'enherbement dans la zone cotonnière Est du Burkina Faso. Cette étude fait ressortir ainsi, l'intérêt d'une lutte intégrée contre l'enherbement dans le contexte de la zone cotonnière Est, car aucune des méthodes de lutte, seule, ne permet de bien maîtriser l'enherbement.

\section{Conclusion}

Les inventaires ont permis de préciser la composition et la diversité floristique des mauvaises herbes des champs de cotonnier dans la zone cotonnière Est du Burkina Faso. La flore adventice de cette zone est principalement dominée par les dicotylédones qui représentent plus de $80 \%$ des familles de mauvaises herbes inventoriées. L'analyse de la fréquence et de l'abondance-dominance a permis d'identifier au total, 12 espèces de mauvaises herbes nuisibles au cotonnier. Le désherbage chimique, le désherbage mécanique et le désherbage manuel sont les moyens de lutte contre ces mauvaises herbes. Ces trois méthodes de lutte complémentaires, constituent la stratégie de gestion de l'enherbement dans les champs de coton. Les difficultés de gestion de l'enherbement sont liées entre autres, au faible niveau d'équipement des producteurs et à la maîtrise insuffisante de la lutte chimique contre les mauvaises herbes, avec notamment, une faible application des herbicides de post-levée. Aussi, faudra-t-il songer à dynamiser le désherbage chimique par des herbicides de post-levée efficaces contre les dicotylédones et les monocotylédones à feuilles larges prédominantes dans la zone d'étude.

\section{CONFLIT D'INTERETS}

Les auteurs déclarent qu'il n'y a aucun conflit d'intérêts lié à cet article.

\section{CONTRIBUTIONS DES AUTEURS}

L'auteur principal $\mathrm{BE}$ a contribué à la conduite de l'étude, notamment la collecte, le traitement des données et la rédaction du manuscrit. BK a assuré la direction scientifique des travaux et a contribué à la rédaction et à la correction du manuscrit. HT a contribué à l'analyse des données et à la correction du manuscrit.

\section{REMERCIEMENTS}

Les auteurs remercient vivement l'Association Interprofessionnelle du Coton du Burkina Faso (AICB) pour le financement de ce travail. Leurs remerciements s'adressent en particulier, à la société cotonnière du Gourma (SOCOMA) pour son appui à la réalisation de l'étude sur le terrain. Ils remercient l'Institut de l'Environnement et de Recherches Agricoles (INERA) pour les infrastructures d'accueil et la logistique dans la réalisation de l'étude.

\section{REFERENCES}

Ahonon BA, Traoré H, Ipou Ipou J. 2018. Mauvaises herbes majeures de la culture de haricot (Phaseolus vulgaris L.) dans la Région du Moronou au Centre-Est de la Côte d'Ivoire. Int. J. Biol. Chem. Sci., 12(1): 310-321. DOI: https://dx.doi.org/10.4314/ijbcs.v12i1.25

Ahanchédé A. 1997. Les mauvaises herbes de la culture cotonnière au sud-Bénin. Bulletin de la Recherche Agronomique, (19): 15-33.

Bassene C, Mbaye MS, Kane A, Diangar S, Noba K. 2012. Flore adventice du maïs (Zea mays L.) dans le sud du Bassin arachidier (Sénégal) : structure et 
nuisibilité des espèces. Journal of Applied Biosciences, 59: 4307- 4320.

Boussim IJ, Yonli D, Guinko S, Salle G. 2011. Etat d'infestation, connaissance endogène et approche systématique des espèces du genre Striga au Burkina Faso. Int. J. Biol. Chem. Sci., 5(4): 1374-1386. DOI

http://dx.doi.org/10.4314/ijbcs.v5i4.4

Boudjedjou L. 2010. Etude de la flore adventice des cultures de la région de la région de Jijel, Mémoire de MAGISTER, Faculté des sciences, Département de Biologie, Université Ferhat Abbas-Setif, p155.

Cardoso GD, Alves PLCA, Severinoa LS, Vale LS. 2011. Critical periods of weed control in naturally green colored cotton BRS Verde. Industrial Crops and Products, 34 (1):1198-1202. DOI: https://doi.org/10.1016/j.indcrop.2011.04 .014

Diouf N, Mbaye MS, Gueye M, Diouf J, Ka SL, Cissoko MK, Diop D, Gueye MF, Dieng B, Camara AA, Noba K. 2020. Degré d'infestation des adventices dans le bassin cotonnier du Sénégal. Int. J. Biol. Chem. Sci., 14(3): 916-927. DOI: https://doi.org/10.4314/ijbcs.v14i3.21.

Ferrell J, MacDonald G, Devkota P. 2020. Weed Management in Cotton". EDIS, (3). DOI: https://doi.org/10.32473/ediswg003-2020.

Fried G. 2010. Variations spatiales et temporelles des communautés adventices des cultures annuelles en France. Acta Botanica Gallica, 157(1): 183-192. DOI: https://doi.org/10.1080/12538078.2010.1 0516198

Garane A, Yonli D, Koussao S, Nikiema J, Traore M, Sawadogo M. 2019. Influence des niveaux de fertilisation sur l'enherbement et l'efficacité des herbicides dans les parcelles d'oignon au centre du Burkina Faso. Int. J. Biol. Chem. Sci., 13(4): 2158-2172. DOI: https://dx.doi.org/10.4314/ijbcs.v13i4.22

Gharde Y, Singh PK, Dubey RP, Gupta PK. 2018. Assessment of yield and economic losses in agriculture due to weeds in
India. Crop Protection, 107(1): 12-18. DOI:

https://doi.org/10.1016/j.cropro.2018.01. 007

Ka SL, Mbaye MS, Gueye M, Camara AA, Dieng B, Noba K. 2019. Flore adventice $\mathrm{du}$ Sorgho (Sorghum bicolor [L.] Moench) en Haute Casamance. Int. J. Biol. Chem. Sci., 13(1): 411-425. DOI: https://dx.doi.org/10.4314/ijbcs.v13i1.32

Ka SL, Gueye M, Kanfany G, Diatta C, Mbaye MS, Noba K. 2020. Dynamique de levée des adventices du sorgho [Sorghum bicolor (L.) Moench] en zone soudanienne humide du Sénégal. Rev. Mar. Sci. Agron. Vét., 8(3): 286-293.

Karar RO, Mohamed BF, Marrs RH. 2005. Factors influencing the weed flora in the Gezira Scheme, Sudan. Weed Research, 45(2): $\quad 121-129 . \quad$ DOI: https://dx.doi.org/10.1111/j.13653180.2004.00441.x

Koulibaly B, Dakouo D, Vognan G. 1998. Contraintes au désherbage des cultures en zone cotonnière ouest du Burkina Faso. 17e Conférence du Columa. Dijon (France). 9-11/12/98. A.N.P.P. 12591266.

Manalil S, Coast O, Werth J, Chauhan BS. 2017. Weed management in cotton (Gossypium hirsutum L.) through weedcrop competition: A review. Crop Protection, 95: 53-59. DOI: http://dx.doi.org/10.1016/j.cropro.2016.0 8.008

Marnotte P, Le Bourgeois T. 2002. Session tropicale du COLUMA 2001. Les mauvaises herbes des cultures tropicales. Phytoma-La Défense des Végétaux (551): 8-12.

MEEVCC, 2018. Annuaire statistique sur l'environnement. Burkina Faso. 255 p.

Oerke EC. 2006. Crop losses to pests. Journal of Agricultural Science, 144(1): 31-43. DOI:

https://doi.org/10.1017/S0021859605005 708

Olina Bassala JP, Wirnkar LV, Marnotte P. 2010. Désherbage chimique et gestion de l'enherbement du cotonnier au Nord- 
Cameroun. In Seiny-Boukar L, Boumard P. (éds). Actes du colloque «Savanes africaines en développement : innover pour durer », 20-23 avril 2009, Garoua, Cameroun. pp.1-9.

Le Bourgeois T. 1993. Les mauvaises herbes dans la rotation cotonnière au Nord Cameroun (Afrique Centrale). Thèse de doctorat, Université de Montpellier II, Sciences et Techniques du Languedoc, France, p.249.

Le Bourgeois T, Marnotte P. 1994. Lutte contre Commelina benghalensis L. : Adventice majeure des cultures cotonnières intensives. Agriculture et Développement, (2): $2 \mathrm{p}$.

Lebreton G, Le Bourgeois T. 2005. Analyse de la flore adventice de la lentille à CilaosRéunion. Saint Pierre : CIRAD-CA, 19 p. Touré A, Ipou Ipou J, Adou Yao CY, Boraud MKN, N'Guessan K. 2008. Diversité floristique et degré d'infestation des mauvaises herbes des agroécosystèmes environnant la forêt classée de Sanaimbo, dans le Centre-Est de la Côte d'Ivoire. Agronomie Africaine, 20(1): 13-22. DOI: http://dx.doi.org/10.4314/aga.v20i1.1732
Traoré H, Maillet J. 1992. Flore adventice des cultures céréalières annuelles du Burkina Faso.

Weed Research, 32(4): 279-293. DOI: https://doi.org/10.1111/j.1365-

3180.1992.tb01888.x

Traoré H, Maillet J. 1998. Mauvaises herbes des cultures céréalières au Burkina Faso. Agriculture et Développement, (20): 4759.

Yonli D, Traore H, Hess DE, Sankara P, Sereme P. 2006. Effect of growth medium, Striga seed burial distance and depth on efficacy of Fusarium isolates to control Striga hermonthica in Burkina Faso. Weed Research, 46(1): 73-81. DOI: https://doi.org/10.1111/j.13653180.2006.00486.x

Yonli D, Traore H, Sereme P, Hess DE, Sankara P, 2010. Pathogenicity of Fusarium isolates to Striga hermonthica in Burkina Faso. Pakistan Journal of Biological Sciences, 13(5): 201-208. DOI: http://dx.doi.org/10.3923/pjbs.2010.201. 208. 